\title{
Análise fitossociológica e estrutura florística de uma floresta estacional decidual
}

\author{
Amauri Bambolim ${ }^{1}$, Abilene Rodrigues Donde ${ }^{1}$, Júlio César Wojciechowsk ${ }^{1}$ \\ ${ }^{1}$ Universidade do Estado de Mato Grosso, Campus Universitário de Alta Floresta, Alta Floresta, Mato Grosso, Brasil. E-mail: \\ amauribambolim@outlook.com, bilene06@gmail.com,cw.julio@gmail.com
}

Recebido: 06/03/2017; Aceito: 18/04/2018

\section{RESUMO}

A análise fitossociológica bem como a composição florística de florestas naturais é de grande valor para compreensão da dinâmica das comunidades florestais. O presente trabalho teve como objetivo analisar uma área de floresta estacional decidual localizada no município de Santa Tereza no estado do Rio Grande do Sul. Foram instaladas 100 parcelas de $10 \times 10$ demarcadas em um hectare no interior da floresta, onde foram medidos e identificados todos os indivíduos com circunferência à altura do peito $(\mathrm{CAP}) \geq 30 \mathrm{~cm}$. O levantamento foi realizado adotando-se o método de parcelas contíguas, seguindo a metodologia de Muller-Dombois e Ellemberg (1974). A densidade total de 875 indivíduos por hectare, sendo 56 espécies, 46 gêneros e 30 famílias. As famílias Fabaceae e Euphorbiaceae apresentaram o maior número de espécies. A espécie Lonchocarpus campestres, apresentou o maior índice de valor de importância (IVI) e maior índice de valor de cobertura (IVC). O índice de diversidade de Shannon (H') foi de 3,34 nats/ind. e o índice de equabilidade de Pielou (J) foi de 0,83. A maior parte dos indivíduos estão em classe de altura entre 8 e $10 \mathrm{~m}$ e classe de diâmetro entre 9 e $15 \mathrm{~cm}$.

Palavras-chave: fitossociologia; estrutura Florestal; espécies arbóreas

\section{Phytosociological analysis and floristic structure of a seasonal deciduous forest}

\begin{abstract}
The phytosociological analysis as well as the floristic composition of natural forests is of great value to understanding the dynamics of forest communities. The present study had as objective to analyze a seasonal deciduous forest area in the municipality of Santa Teresa, Rio Grande do Sul State, Brazil. Have been installed 100 plots $10 \times 10$ demarcated in a hectare within the forest where they were measured and identified all individuals with Circumference at breast height $(\mathrm{CBH}) \geq 30 \mathrm{~cm}$. The survey was conducted by adopting the method of contiguous parcels, following the methodology of Mueller-Dombois and Ellemberg (1974). The total density was 875 individuals per hectare, which belong 56 species, 49 genera and 30 families. The families Fabaceae and Euphorbiaceae showed the largest number of species. The species Lonchocarpus campestres presented the biggest importance value index (IVI) and higher value index (CVI). The Shannon diversity index (H ') was 3.34 nats/ind. and the Pielou evenness index (J) was 0.83. Most individuals are in high class between 8 and $10 \mathrm{~m}$ and a diameter between 9 and $15 \mathrm{~cm}$.
\end{abstract}

Key words: phytosociology; forest structure; tree species. 


\section{Introdução}

O conhecimento da flora e seus padrões estruturais é de grande importância para que se possa avançar na elaboração de planos de manejos sustentáveis com o objetivo de conservar as florestas e garantir seu uso para as gerações futuras (GONÇALVES, 2015) A Floresta Estacional Decidual se apresenta como a principal tipologia florestal encontrada no Rio Grande do Sul e tem sido ameaçada pela expansão da agricultura e áreas urbanas (CALLEGARO, et al. 2014).

A realização de uma avaliação da estrutura vertical e horizontal da floresta, além do reconhecimento das espécies presentes no local torna-se imprescindível para caracterizar o comportamento da vegetação de uma determinada região. A estrutura horizontal analisa densidade, dominância, frequência e importância das espécies e a estrutura vertical permite avaliar o desenvolvimento da floresta com base nos diferentes estratos arbóreos (GUEDES; KRUPEK, 2016).

Outro aspecto importante na avaliação de formações florestais é a diversidade. Definir a diversidade é complexo pois há grande variação e abundância de espécies. O índice de diversidade de Shannon é um dos mais usados pelo fato de combinar o número de espécies presentes e a densidade relativa da espécie em um único valor (HACK, et al. 2005).

Com o intuito de aumentar o conhecimento a respeito dos remanescentes de floresta estacional decidual e contribuir para a elaboração de projetos de recuperação e preservação destes remanescentes o presente trabalho teve como objetivo realizar um levantamento florístico e fitossociológico de um fragmento de floresta estacional decidual no município de Santa Tereza Rio Grande do Sul (RS).

\section{Material e Métodos}

O município de Santa Tereza, localizado na região nordeste do Rio Grande do Sul sob as coordenadas $29^{\circ}$

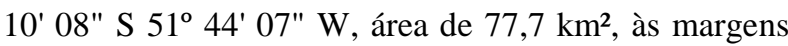
do rio Taquari com altitude $87 \mathrm{~m}$, é rodeado por elevações de terreno montanhoso que atingem até $550 \mathrm{~m}$ de altitude, formando vales. O clima local é do tipo Cfa, (subtropical temperado úmido).

O levantamento foi realizado adotando-se o método de parcelas contíguas, seguindo a metodologia de Muller-Dombois e Ellemberg (1974). Foram estabelecidas 100 unidades amostrais de 10 x $10 \mathrm{~m}$ (100 $\mathrm{m}^{2}$ ) totalizando uma área de um hectare, onde foram registradas, identificadas e mensuradas todas as árvores com circunferência a altura do peito $(C A P) \geq 30 \mathrm{~cm}$. Em fichas específicas de campo, anotou-se a espécie botânica, altura total e o ponto de inversão morfológica obtida por hipsómetro Vertex II e o CAP, utilizando uma fita métrica. Para determinar a estrutura horizontal da comunidade, foram determinados os seguintes parâmetros: densidade, dominância e frequência (tanto relativa como absoluta), área basal, valor de cobertura (IVC) e índice de valor de importância (IVI).

Para o cálculo da diversidade utilizou-se o índice de diversidade de Shannon (H'). Quanto maior for o valor de H', maior será a diversidade florística da população em estudo. Também foi utilizado o índice de Pielou (J) que determina o padrão de distribuição dos indivíduos variando entre 0 e 1 , onde 1 representa a máxima diversidade dentro da comunidade. $\mathrm{O}$ cálculo dos dados e a representação gráfica foi realizada com o software Microsoft Excel 2013 e R Core Team (2016).

\section{Resultados e Discussão}

$\mathrm{Na}$ área estudada foram catalogados 875 indivíduos pertencentes a 30 famílias, 46 gêneros e 56 espécies (Tabela 1). Resultado semelhante foi encontrado por Hack et al. (2005), para o número de família e espécies 28 e 54 respectivamente.

A família que apresentou maior riqueza florística foi a Fabaceae com 6 espécies seguida da família Euphorbiaceae com 4 espécies Figura 1.

As espécies que mais se destacaram foram Lonchocarpus campestres (128), Solanum pseudoquina (77), Solanum sanctae-catharinae (63), Cupania vernalis (52), Ficus luschnathiana (52), e Myrocarpus frondosus (50), Figura 2. Estas espécies foram responsáveis por 48,22\% dos indivíduos amostrados.

A área amostrada apresentou uma densidade absoluta de 875 indivíduos por hectare. Tabela 2 . Balbinot et al. (2016), encontraram 842 indivíduos em área de Floresta Estacional Decidual no Estado do Rio Grande do Sul.

A espécie que apresentou maior índice de valor de importância (IVI), Lonchocarpus campestres (30,7), também apresentou o maior índice de valor de cobertura (IVC) com índice de 27,11e maior Dominância Absoluta e Relativa; a área basal (G) das árvores mensuradas foi de $15,18 \mathrm{~m}^{2} /$ ha. (Tabela 2). Resultado encontrado por Haidar et al. (2013) em floresta decidual revelou área basal de $33,15 \mathrm{~m}$ por hectare.

A diversidade calculada pelo índice de Shannon (H'), que indica a diversidade da floresta apresentou valor de 3,34 nats. Ind. Resultado semelhante ao encontrado por Maboni et al. (2015), que encontraram valor de 3,411nats. Ind. para o índice de Shannon. O índice de equabilidade de Pielou (J) de 0,83 indicou grande uniformidade na composição das parcelas. Corsini, et al. (2014) encontraram índice de equabilidade de Pielou de 0,85, semelhante ao da presente pesquisa. 
Tabela 1. Família, nome científico e nome vulgar de uma área de floresta estacional decidual no município de Santa Tereza RS.

\begin{tabular}{|c|c|}
\hline Família/espécie & Nome Popular \\
\hline \multicolumn{2}{|l|}{ Fabaceae } \\
\hline Lonchocarpus campestris Mart. ex Benth. & Pau-canzil \\
\hline Myrocarpus frondosus Allemão & Cabriúva \\
\hline Machaerium stipitatum (DC.) Vogel & Canela-do-brejo \\
\hline Parapiptadenia rigida (Benth.) Brenan & Angico-vermelho \\
\hline Machaerium paraguariense Hassl. & Farinha Seca \\
\hline Albizia edwallii (Hoehne) Barneby \& J.Grimes & Angico-pururuca \\
\hline \multicolumn{2}{|l|}{ Moraceae } \\
\hline Ficus luschnathiana (Miq.) Miq. & Figueira-do-mato \\
\hline Sorocea bonplandii (Baill.) W.C. Burger, Lanjouw \& Boer & Cincho \\
\hline \multicolumn{2}{|l|}{ Solanaceae } \\
\hline Solanum pseudoquina A. St.-Hill. & Peloteira \\
\hline Solanum sanctaecatharinae Dunal & Joá-manso \\
\hline Cestrum L. & Coerana \\
\hline \multicolumn{2}{|l|}{ Mytacceae } \\
\hline Myrcianthes pungens (O.Berg) D. Legrand & Guabijú \\
\hline Campomanesia xanthocarpa O.Berg & Guabiroba \\
\hline Blepharocalyx salicifolius (Kunth) O.Berg & Murta \\
\hline \multicolumn{2}{|l|}{ Sapindaceae } \\
\hline Cupania vernalis Cambess. & Camboatá-vermelho \\
\hline Matayba elaeagnoides Radlk. & Camboatá-branco \\
\hline Allophylus edulis A.St.-Hil., Cambess. \& A. Juss. & Chal-chal \\
\hline \multicolumn{2}{|l|}{ Malvaceae } \\
\hline Luehea divaricata Mart. \& Zucc. & Açoita-cavalo \\
\hline \multicolumn{2}{|l|}{ Ebenaceae } \\
\hline Diospyros inconstans Jacq. & Fruta-de-jacú \\
\hline \multicolumn{2}{|l|}{ Apocynaceae } \\
\hline Aspidosperma parvifolium A. DC. & Guatambú \\
\hline \multicolumn{2}{|l|}{ Sapotacea } \\
\hline Chrysophyllum marginatum (Hook. \& Arn.) Radlk. & Aguaí-leiteiro \\
\hline \multicolumn{2}{|l|}{ Euphorbiaceae } \\
\hline Manihot grahamii Hook. & Mandiocão \\
\hline Sebastiania commersoniana (Baill.) L.B. Sm. \& Downs & Braquilho-comum \\
\hline Sapium glandulosum (L.) Morong & Pau-leiteiro \\
\hline Sebastiania brasiliensis Spreng. & Branquilho-leiteiro \\
\hline \multicolumn{2}{|l|}{ Salicaceae } \\
\hline Banara tomentosa Clos & Guaçatunga-branca \\
\hline Xylosma pseudosalzmanii Sleumer & Espinho-judeu \\
\hline Casearia sylvestris $\mathrm{Sw}$ & chá-de-bugre \\
\hline \multicolumn{2}{|l|}{ Lauraceae } \\
\hline Ocotea puberula (Rich.) Nees & Canela-guaicá \\
\hline Nectandra megapotamica (Spreng.) Mez & Canela-preta \\
\hline Ocotea silvestris Vattimo-Gil & Canela-ferrugem \\
\hline \multicolumn{2}{|l|}{ Rubiaceae } \\
\hline Randia ferox (Cham. \& Schltdl.) DC. & Limoeiro-do-mato \\
\hline \multicolumn{2}{|l|}{ Rutaceae } \\
\hline Helietta apiculata Benth. & Canela-de-veado \\
\hline Zanthoxylum fagara (L.) Sarg. & Coentrilho \\
\hline Zanthoxylum rhoifolium Lam. & Mamica-de-cadela \\
\hline \multicolumn{2}{|l|}{ Escalloniaceae } \\
\hline Escallonia bifida Link \& Otto & Canudo-de-pito \\
\hline \multicolumn{2}{|l|}{ Caricaceae } \\
\hline Vasconcellea quercifolia A. St.-Hil. & Mamoeiro-do-mato \\
\hline \multicolumn{2}{|l|}{ Boraginaceae } \\
\hline Cordia americana (L.) Gottshling \& J.E.Mill. & Guajuvira \\
\hline Cordia ecalyculata Vell. & Louro-mole \\
\hline \multicolumn{2}{|l|}{ Meliaceae } \\
\hline Cabralea canjerana (Vell.) Mart. & Cangerana \\
\hline
\end{tabular}




\begin{tabular}{|c|c|}
\hline Trichilia claussenii C.DC. & Catiguá-vermelho \\
\hline \multicolumn{2}{|l|}{ Anonaceae } \\
\hline Annona rugulosa (Schltdl.) H.Rainer & Araticum-quaresma \\
\hline Annona neosalicifolia H.Rainer & Araticum-salso \\
\hline \multicolumn{2}{|l|}{ Bignoniaceae } \\
\hline Tecoma stans (L.) Juss. ex Kunth & Caroba-louca \\
\hline \multicolumn{2}{|l|}{ Loganiaceae } \\
\hline Strychnos brasiliensis (Spreng.) Mart. & Anzol-de-lontra \\
\hline \multicolumn{2}{|l|}{ Simaroubaceae } \\
\hline Picrasma crenata (Vell.) Engl. & Pau-amargo \\
\hline \multicolumn{2}{|l|}{ Cannabaceae } \\
\hline Trema micrantha (L.) Blume & Grandiúva \\
\hline Celtis iguanaea (Jacq.) Sarg. & Espoão-de-galo \\
\hline \multicolumn{2}{|l|}{ Rosaceae } \\
\hline Prunus myrtifolia (L.) Urb. & Pessegueiro-do-mato \\
\hline \multicolumn{2}{|l|}{ Rhamaceae } \\
\hline Hovenia dulcis Thunb. & Uva-do-japão \\
\hline \multicolumn{2}{|l|}{ Arecaceae } \\
\hline Syagrus romanzoffiana (Cham.) Glassman & Jerivá \\
\hline \multicolumn{2}{|l|}{ Cardiopteridaceae } \\
\hline Citronella paniculata (Mart.) Howard & Gongonha \\
\hline \multicolumn{2}{|l|}{ Phytolaccaceae } \\
\hline Phytolacca dioica $\mathrm{L}$. & Umbú \\
\hline \multicolumn{2}{|l|}{ Primulaceae } \\
\hline Myrsine umbellata Mart. & Pororocão \\
\hline \multicolumn{2}{|l|}{ Nyctaginaceae } \\
\hline Pisonia ambigua Heimerl & Maria-mole \\
\hline
\end{tabular}

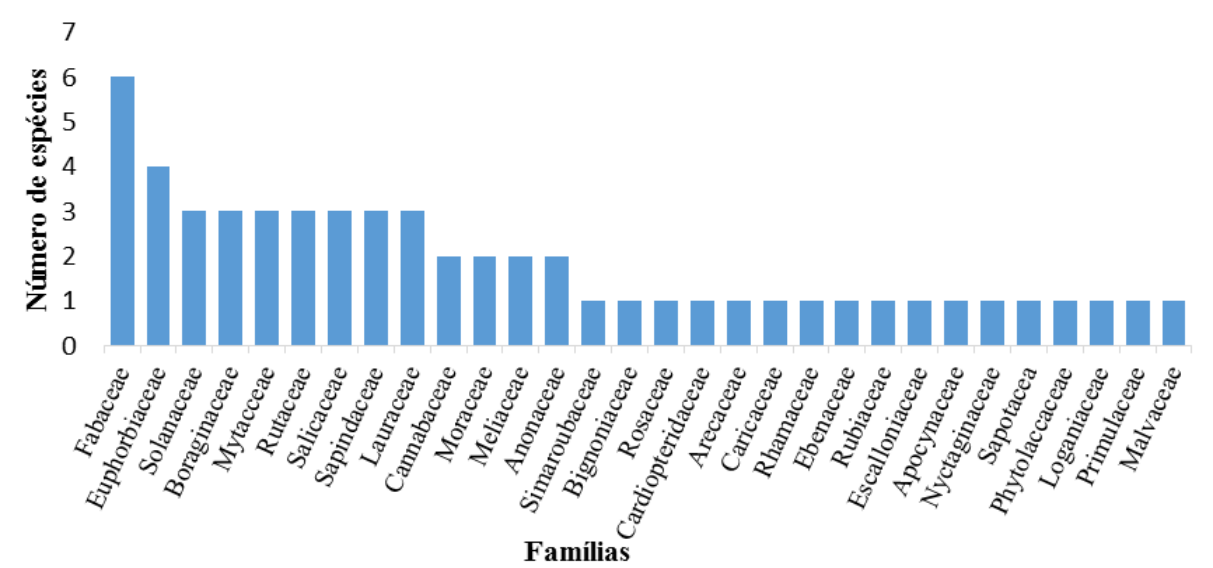

Figura 1. Número de espécies por família inventariadas em floresta estacional decidual no município de Santa Tereza, RS.

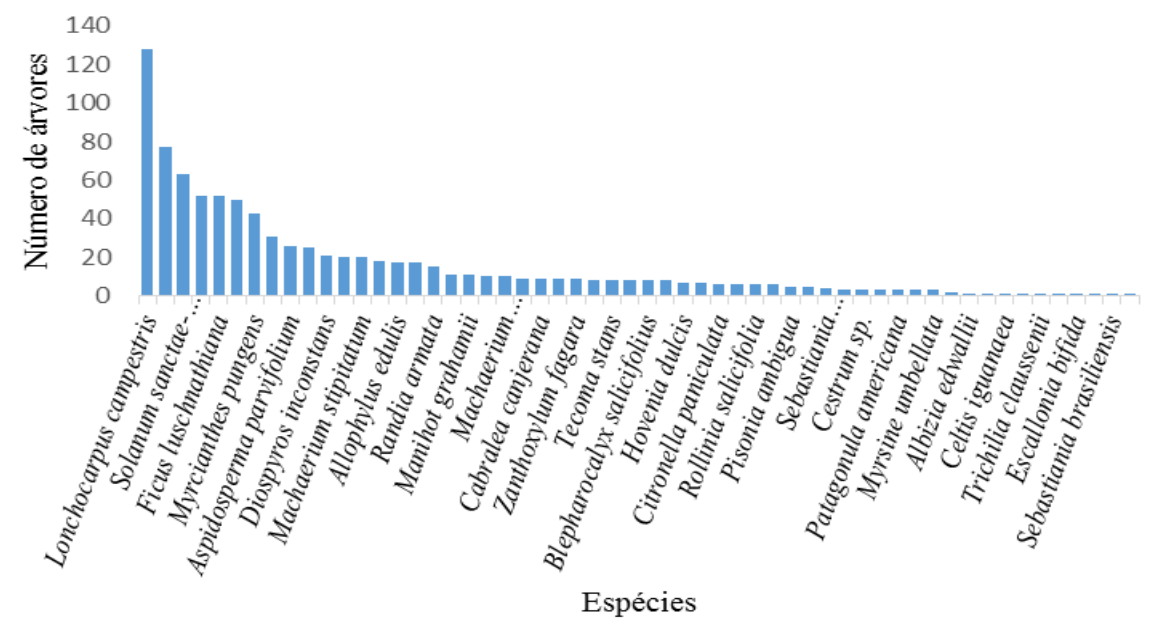

Figura 2. Número de indivíduos amostrados por espécie no município de Santa Tereza, RS. 
Tabela 2. Parâmetros estruturais da vegetação no município de Santa Tereza RS.

\begin{tabular}{|c|c|c|c|c|c|c|c|c|c|c|c|c|}
\hline Nome Científico & Nome Popular & $\mathbf{N}$ & G & DA & DR & DoA & DoR & FA & FR & IVC & IVI & IVIA \\
\hline Albizia edwallii & Angico-pururuca & 1 & 0,0076 & 1 & 0,11 & 0,008 & 0,05 & 100 & 4,5 & 0,16 & 4,7 & 4,7 \\
\hline Allophylus edulis & Chal-chal & 17 & 0,3028 & 17 & 1,94 & 0,303 & 1,99 & 40 & 1,8 & 3,94 & 5,7 & 7,8 \\
\hline Aspidosperma parvifolium & Guatambú & 26 & 0,4891 & 26 & 2,97 & 0,489 & 3,22 & 10 & 0,5 & 6,19 & 6,6 & 10,0 \\
\hline Banara tomentosa & Guaçatunga-branca & 5 & 0,0995 & 5 & 0,57 & 0,099 & 0,66 & 90 & 4,1 & 1,23 & 5,3 & 5,9 \\
\hline Blepharocalyx salicifolius & Murta & 8 & 0,1855 & 8 & 0,91 & 0,186 & 1,22 & 10 & 0,5 & 2,14 & 2,6 & 3,5 \\
\hline Cabralea canjerana & Canjerana & 9 & 0,1741 & 9 & 1,03 & 0,174 & 1,15 & 10 & 0,5 & 2,18 & 2,6 & 3,7 \\
\hline Campomanesia xanthocarpa & Guabiroba & 8 & 0,1073 & 8 & 0,91 & 0,107 & 0,71 & 40 & 1,8 & 1,62 & 3,4 & 4,5 \\
\hline Carica quercifolia & Mamoeiro-do-mato & 8 & 0,1407 & 8 & 0,91 & 0,141 & 0,93 & 40 & 1,8 & 1,84 & 3,7 & 4,8 \\
\hline Casearia sylvestris & Chá-de-bugre & 17 & 0,2006 & 17 & 1,94 & 0,201 & 1,32 & 10 & 0,5 & 3,26 & 3,7 & 5,9 \\
\hline Celtis iguanaea & Esporão-de-galo & 1 & 0,0121 & 1 & 0,11 & 0,012 & 0,08 & 40 & 1,8 & 0,19 & 2,0 & 2,2 \\
\hline Cestrum sp. & Coerana & 3 & 0,0281 & 3 & 0,34 & 0,028 & 0,19 & 50 & 2,3 & 0,53 & 2,8 & 3,2 \\
\hline Chrysophyllum marginatum & Aguaí-leiteiro & 9 & 0,1266 & 9 & 1,03 & 0,127 & 0,83 & 90 & 4,1 & 1,86 & 5,9 & 7,3 \\
\hline Citronella paniculata & Gongonha & 6 & 0,0798 & 6 & 0,69 & 0,080 & 0,53 & 10 & 0,5 & 1,21 & 1,7 & 2,4 \\
\hline Cordia ecalyculata & Louro-mole & 6 & 0,0674 & 6 & 0,69 & 0,067 & 0,44 & 30 & 1,4 & 1,13 & 2,5 & 3,1 \\
\hline Cupania vernalis & Camboatá-vermelho & 52 & 0,5599 & 52 & 5,94 & 0,560 & 3,69 & 60 & 2,7 & 9,63 & 12,3 & 19,1 \\
\hline Diospyros inconstans & Fruta-de-jacú & 21 & 0,3112 & 21 & 2,40 & 0,311 & 2,05 & 60 & 2,7 & 4,45 & 7,2 & 9,6 \\
\hline Escallonia bifida & Canudo-de-pito & 1 & 0,0081 & 1 & 0,11 & 0,008 & 0,05 & 80 & 3,6 & 0,17 & 3,8 & 3,8 \\
\hline Ficus luschnathiana & Figueira-do-mato & 52 & 1,5616 & 52 & 5,94 & 1,562 & 10,29 & 50 & 2,3 & 16,23 & 18,5 & 24,1 \\
\hline Helietta apiculata & Canela-de-veado & 11 & 0,1238 & 11 & 1,26 & 0,124 & 0,82 & 50 & 2,3 & 2,07 & 4,3 & 5,8 \\
\hline Hovenia dulcis & Uva-do-japão & 7 & 0,0837 & 7 & 0,80 & 0,084 & 0,55 & 10 & 0,5 & 1,35 & 1,8 & 2,7 \\
\hline Lonchocarpus campestris & Pau-canzil & 128 & 1,8949 & 128 & 14,63 & 1,895 & 12,48 & 80 & 3,6 & 27,11 & 30,7 & 45,9 \\
\hline Luehea divaricata & Açoita-cavalo & 20 & 0,3241 & 20 & 2,29 & 0,324 & 2,14 & 100 & 4,5 & 4,42 & 8,9 & 11,4 \\
\hline Machaerium paraguariense & Canela-do -brejo & 10 & 0,2666 & 10 & 1,14 & 0,267 & 1,76 & 40 & 1,8 & 2,90 & 4,7 & 5,9 \\
\hline Machaerium stipitatum & Canela-do-brejo & 20 & 0,4105 & 20 & 2,29 & 0,411 & 2,70 & 50 & 2,3 & 4,99 & 7,3 & 9,4 \\
\hline Manihot grahamii & Mandiocão & 11 & 0,1417 & 11 & 1,26 & 0,142 & 0,93 & 70 & 3,2 & 2,19 & 5,4 & 6,6 \\
\hline Matayba elaeagnoides & Camboatá-branco & 31 & 0,8126 & 31 & 3,54 & 0,813 & 5,35 & 10 & 0,5 & 8,90 & 9,3 & 13,0 \\
\hline Morta & Morta & 10 & 0,1893 & 10 & 1,14 & 0,189 & 1,25 & 20 & 0,9 & 2,39 & 3,3 & 4,5 \\
\hline Myrcianthes pungens & Guabijú & 43 & 1,1839 & 43 & 4,91 & 1,184 & 7,80 & 30 & 1,4 & 12,71 & 14,1 & 19,0 \\
\hline Myrocarpus frondosus & Cabriúva & 50 & 1,2583 & 50 & 5,71 & 1,258 & 8,29 & 10 & 0,5 & 14,00 & 14,5 & 19,9 \\
\hline Myrsine umbellata & Capororocão & 3 & 0,0915 & 3 & 0,34 & 0,091 & 0,60 & 10 & 0,5 & 0,95 & 1,4 & 1,7 \\
\hline Nectandra megapotamica & Canela-preta & 3 & 0,0976 & 3 & 0,34 & 0,098 & 0,64 & 70 & 3,2 & 0,99 & 4,2 & 4,5 \\
\hline Ocotea puberula & Canela-guaicá & 6 & 0,2006 & 6 & 0,69 & 0,201 & 1,32 & 60 & 2,7 & 2,01 & 4,7 & 5,5 \\
\hline Ocotea silvestris & Canela ferrugem & 9 & 0,1222 & 9 & 1,03 & 0,122 & 0,81 & 10 & 0,5 & 1,83 & 2,3 & 3,3 \\
\hline Parapiptadenia rigida & Angico-vermelho & 1 & 0,0844 & 1 & 0,11 & 0,084 & 0,56 & 100 & 4,5 & 0,67 & 5,2 & 5,2 \\
\hline Patagonula americana & Guajuvira & 3 & 0,0266 & 3 & 0,34 & 0,027 & 0,18 & 50 & 2,3 & 0,52 & 2,8 & 3,1 \\
\hline Phytolacca dioica & Umbú & 1 & 0,0081 & 1 & 0,11 & 0,008 & 0,05 & 30 & 1,4 & 0,17 & 1,5 & 1,6 \\
\hline Picrasma crenata & Pau-amargo & 8 & 0,0868 & 8 & 0,91 & 0,087 & 0,57 & 20 & 0,9 & 1,49 & 2,4 & 3,5 \\
\hline Pisonia ambigua & Maria-mole & 5 & 0,0488 & 5 & 0,57 & 0,049 & 0,32 & 10 & 0,5 & 0,89 & 1,3 & 2,0 \\
\hline Prunus myrtifolia & Pessegueiro-do-mato & 3 & 0,0302 & 3 & 0,34 & 0,030 & 0,20 & 30 & 1,4 & 0,54 & 1,9 & 2,3 \\
\hline Randia armata & Limoeiro-do-mato & 15 & 0,2997 & 15 & 1,71 & 0,300 & 1,97 & 20 & 0,9 & 3,69 & 4,6 & 6,5 \\
\hline Rollinia rugulosa & Araticum-quaresma & 3 & 0,0576 & 3 & 0,34 & 0,058 & 0,38 & 40 & 1,8 & 0,72 & 2,5 & 2,9 \\
\hline Rollinia salicifolia & Araticum-salso & 6 & 0,0696 & 6 & 0,69 & 0,070 & 0,46 & 20 & 0,9 & 1,14 & 2,0 & 2,7 \\
\hline Sapium glandulatum & Pau-leiteiro & 1 & 0,0121 & 1 & 0,11 & 0,012 & 0,08 & 40 & 1,8 & 0,19 & 2,0 & 2,2 \\
\hline Sebastiania brasiliensis & Branquilho-leiteiro & 1 & 0,0076 & 1 & 0,11 & 0,008 & 0,05 & 40 & 1,8 & 0,16 & 2,0 & 2,0 \\
\hline Sebastiania commersoniana & Branquilho-comum & 4 & 0,0821 & 4 & 0,46 & 0,082 & 0,54 & 40 & 1,8 & 1,00 & 2,8 & 3,0 \\
\hline
\end{tabular}




\begin{tabular}{|c|c|c|c|c|c|c|c|c|c|c|c|c|}
\hline Solanum pseudoquina & Peloteira & 77 & 0,7964 & 77 & 8,80 & 0,796 & 5,25 & 80 & 3,6 & 14,05 & 17,7 & 23,7 \\
\hline Solanum sanctae-catharinae & Joá-manso & 63 & 0,8923 & 63 & 7,20 & 0,892 & 5,88 & 40 & 1,8 & 13,08 & 14,9 & 21,6 \\
\hline Sorocea bonplandii & Cincho & 25 & 0,3179 & 25 & 2,86 & 0,318 & 2,09 & 10 & 0,5 & 4,95 & 5,4 & 8,7 \\
\hline Strychnos brasiliensis & Anzol-de-lontra & 1 & 0,0121 & 1 & 0,11 & 0,012 & 0,08 & 50 & 2,3 & 0,19 & 2,5 & 2,6 \\
\hline Syagrus romanzoffiana & Jerivá & 7 & 0,0653 & 7 & 0,80 & 0,065 & 0,43 & 10 & 0,5 & 1,23 & 1,7 & 2,6 \\
\hline Tecoma stans & Caroba-louca & 8 & 0,1002 & 8 & 0,91 & 0,100 & 0,66 & 20 & 0,9 & 1,57 & 2,5 & 3,4 \\
\hline Trema micrantha & Grandiúva & 1 & 0,0703 & 1 & 0,11 & 0,070 & 0,46 & 40 & 1,8 & 0,58 & 2,4 & 2,5 \\
\hline Trichilia claussenii & Catiguá-vermelho & 1 & 0,0424 & 1 & 0,11 & 0,042 & 0,28 & 30 & 1,4 & 0,39 & 1,8 & 1,8 \\
\hline Xylosma pseudosalzmannii & Espinho-judeu & 18 & 0,2007 & 18 & 2,06 & 0,201 & 1,32 & 10 & 0,5 & 3,38 & 3,8 & 5,8 \\
\hline Zanthoxylum fagara & Coentrilho & 9 & 0,1761 & 9 & 1,03 & 0,176 & 1,16 & 30 & 1,4 & 2,19 & 3,5 & 4,4 \\
\hline Zanthoxylum rhoifolium & Mamica-de-cadela & 2 & 0,0292 & 2 & 0,23 & 0,029 & 0,19 & 10 & 0,5 & 0,42 & 0,9 & 1,2 \\
\hline TOTAL & & & 15,179 & 875 & 100 & 15,18 & 100 & & 100 & 200 & 300 & 400 \\
\hline
\end{tabular}

Observação: $\mathrm{N}=$ número de indivíduos $\mathrm{G}=$ área basal $\mathrm{DA}=$ densidade absoluta $\mathrm{DR}=$ densidade relativa $\mathrm{FA}=$ frequência absoluta $\mathrm{FR}=$ frequência relativa DoA= dominância absoluta DoR= dominância relativa IVC= índice de valor de cobertura IVI= índice de valor de importância.

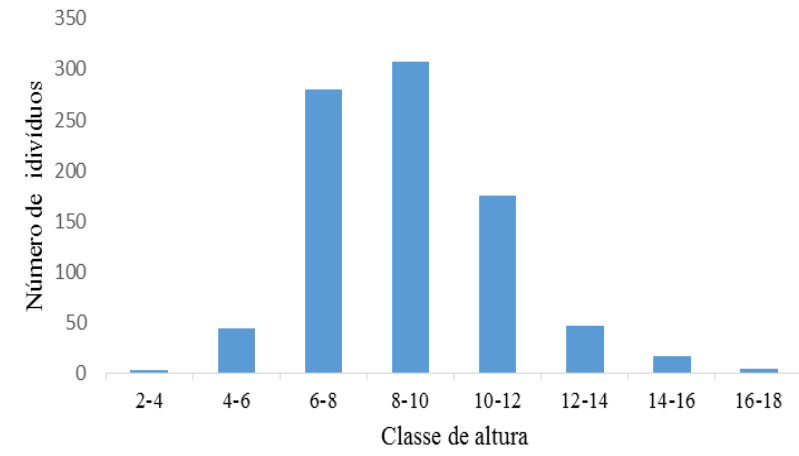

Figura 3. Distribuição do número de indivíduos por classe de altura no município de Santa Tereza, RS.

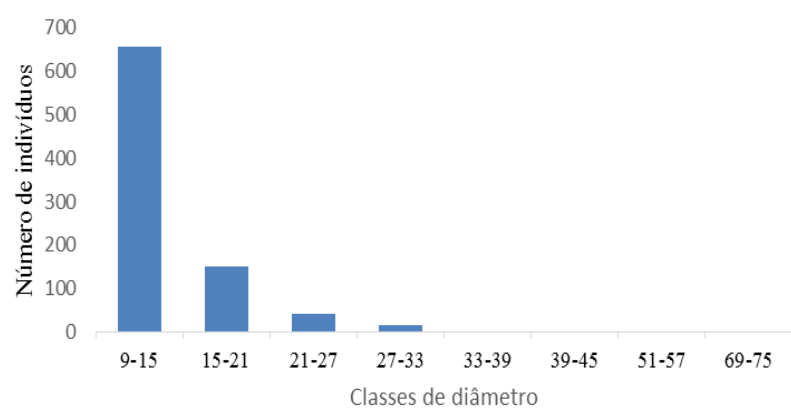

Figura 4. Distribuição do número de indivíduos por classe de diâmetro no município de Santa Tereza RS.

A distribuição vertical dos indivíduos indicou que $308(35,2 \%)$ árvores estão entre 8 e 10 metros de altura (Figura 3). A altura máxima foi atingida pela espécie Parapiptadenia rígida $(17,4 \mathrm{~m})$ e a altura mínima de 2,30 m, da espécie Manihot grahamii.

Com relação ao diâmetro a classe que mais se destacou foi entre 9 e $15 \mathrm{~cm}$, com $75 \%$ dos indivíduos mensurados (656) árvores (Figura 4). Os indivíduos que apresentaram maior diâmetro foram Myrsine umbellata com 73,2 cm e Ficus luschnathiana com 54,43 cm; os menores diâmetros foram das espécies Ficus luschnathiana com $9,55 \mathrm{~cm}$ e Strychnos brasiliensis com $9,86 \mathrm{~cm}$.
A distribuição das classes de diâmetro ficou próxima do "J" invertido ou exponencial negativa, onde existe uma predominância dos indivíduos jovens nas primeiras classes diamétricas, observando decréscimo à medida que se avança para as últimas classes, sugerindo-se que a floresta passa por processo de regeneração e possui potencial de estoque futuro.

\section{Conclusões}

O presente estudo revelou que a área pesquisada apresenta alta diversidade florística e elevada densidade. As famílias Fabaceae e Euphorbiaceae foram as que apresentaram maior número de espécies.

A espécie Lonchocarpus campestres apresentou maior IVC e IVI. As espécies mais abundantes na floresta estacional decidual de Santa Tereza foram Lonchocarpus campestres, Solanum pseudoquina, Solanum sanctae-catharinae, Cupania vernalis, Ficus luschnathiana e Myrocarpus frondosus.

\section{Referências Bibliográficas}

BALBINOT, R.; LAMBRECHT, F. R.; BREUNIG, F. M.; TRAUTENMÜLLER, J. W.; GALVÃO, L. S.; DENARDI, L.; VENDRUSCOLO, R. Análise fitossociológica de um fragmento de Floresta Estacional Decidual: Parque Estadual do Turvo, RS. Revista Pesquisa Florestal Brasileira, Colombo-PR, v. 36, n. 86, p. 103-113, 2016.

CALlEGARO, R. M.; ARAÚJO, M. A.; LONGHI, S. L. Fitossociologia de agrupamentos em Floresta Estacional Decidual no Parque Estadual Quarta Colônia, Agudo - RS. Agrária - Revista Brasileira de Ciências Agrárias, RecifePE, v. 9, n. 4, p. 590-598, 2014.

CORSINI, C. R.; SCOLFORO, J. R. S.; OLIVEIRA, A. D.; MELlO, J. M.; MACHADO, E. L. M. Diversidade e similaridade de fragmentos florestais nativos situados na região nordeste de Minas Gerais. Cerne, Lavras-MG, v. 20, n. 1, p. 1-10, 2014 
GONÇALVES, T. S. A floresta estacional decidual no Brasil: distribuição geográfica e influência dos aspectos pedogeomorfológicos na vegetação. Revista Monografias Ambientais - REMOA, Santa Maria-RS, v.14, n.1, p.144$153,2015$.

GUEDES, J.; KRUPEK, R. A. Florística e fitossociologia do componente arbóreo de um fragmento de floresta ombrófila densa do estado de São Paulo. Revista Acta Biológica Catarinense, Joinville-SC, v. 3, n. 1, p. 12-24, 2016.

HAIDAR, R. F.; FAGG, J. M. F.; PINTO, J. R. R.; DIAS, R. R.; DAMASCO, G.; SILVA, L. C. R.; FAGG, C. W. Florestas estacionais e áreas de ecótono no estado do Tocantins, Brasil: parâmetros estruturais, classificação das fitofisionomias florestais e subsídios para conservação. Acta Amazonica, Manaus-AM, v. 43, n. 3, p. 261-290, 2013.

MABONI, C. A.; MACHADO, M. A.; LONGHI, S. J.; ROVEDDER, A. P.; VOLPATO, S. M. S.; D’ AVILA, M. C. A.S.; DA SILVA, T. T. Análise de agrupamentos em remanescente de floresta estacional decidual. Revista Ciência Florestal, Santa Maria-RS, v. 25, n. 3, p. 781-789, 2015.

R CORE TEAM. R: A language and environment for statistical computing. Vienna, Austria: $\mathrm{R}$ Foundation for Statistical Computing, 2016. Disponível em: <https://www.Rproject.org/>. Acesso em: 14 dez. 2016. 